\title{
Evaluating Use of Higher Dose Oxybutynin in Combination with \\ Desmopressinfor Refractory Nocturnal Enuresis
}

Aaron Berkenwald MS4, Jacqueline Pires MS3, Pamela Ellsworth MD

\section{UMASS.}

\section{Background}

Nocturnal Enuresis (NE) is a common pediatric condition with an overall prevalence of $15-20 \%$ at 5 years of age, with a spontaneous resolution of about $15 \%$ per year. $2 \%$ Of children age 15 still suffer from the condition and limited treatment options exist. Behavioral therapy achieves success in nearly $3 / 4$ of children, but many families prefer medical intervention, especially in older children. Pharmacologic therapies including Desmopressin (DDAVP) or Imipramine are effective in $40-50 \%$ of children. However Imipramine has serious safety concerns. DDAVP in combination with a fixed dose anticholinergic has been shown to be useful in individuals who fail DDAVP alone, but still fails to achieve success rates greater than $60 \%$. We hypothesize that by titrating up the dose of Oxybutynin in combination with DDAVP in patients who fail initial monotherapy, we will achieve higher rates of success with limited additional adverse events. We will also record patient demographics, associated symptoms and co-morbidities to determine if we can predict treatment success in patient subgroups.

\section{Treatment Options \& Success Rates}

$>$ Bedwetting Alarm (Behavioral Therapy) 75\% $>$ DDAVP 50\%

$>$ Imipramine $\sim 40 \%$

$>$ DDAVP + Fixed Dose Anticholinergic 44-57\%

\section{Objectives}

\section{Primary Objective}

To investigate the efficacy of combination therapy (DDAVP + escalating dose Oxybutynin) in children with nocturnal enuresis refractory to maximal dose DDAVP

\section{Secondary Objectives}

$>$ Identify risk factors for monotherapy refractory NE Identify factors that predict success with combination therapy

\section{Methods}

IRB approved retrospective analysis of NE patients, ages 7-18 seen at the UMMS Pediatric Urology Clinic from Nov, 2013 Dec, 2014.

\section{Inclusion Criteria}

Diagnosis of Primary Nocturnal Enuresis or

Nocturnal Enuresis with Controlled ${ }^{*}$ or

resolved Daytime Voiding Symptoms (CDVS)

$\checkmark$ Treatment with at least 1 dose DDAVP

At least one follow-up visit in clinic "controlled with daytime use anticholinergic

\section{Exclusion Criteria}

Active daytime incontinence

Dysfunctional voiding requiring PT

Neurogenic bladder

Noncompliance with therapy

Failure to attend follow-up

\section{Treatment Overview}

Standard Bladder Education

Associated Factors and Demographics Documented

\section{- Initial Monotherapy with DDAVP}

Starting Dose 0.2 or $0.4 \mathrm{mg}$ DDAVP with $0.2 \mathrm{mg}$ increase

Follow-up prior to beginning combination therapy

Low Dose Combination Therapy (LDCT

Advanced Dose Combination Therapy (ADCT) Starting Dose 0.6mg DDAVP $+7.5 \mathrm{mg}$ Oxybutynin IR with 2.5mg increase in Oxybutynin after 2-weeks withou

\section{End Points}

Success on any medication was defined as 14 consecutive nights without bed-wetting event Telephone contact occurred during the dose titration interval until:

1. Effective dose had been achieved

2. Maximal doses of DDAVP and oxybutynin had been tried

- Adverse events were solicited verbally during the phone conversation or in the clinic

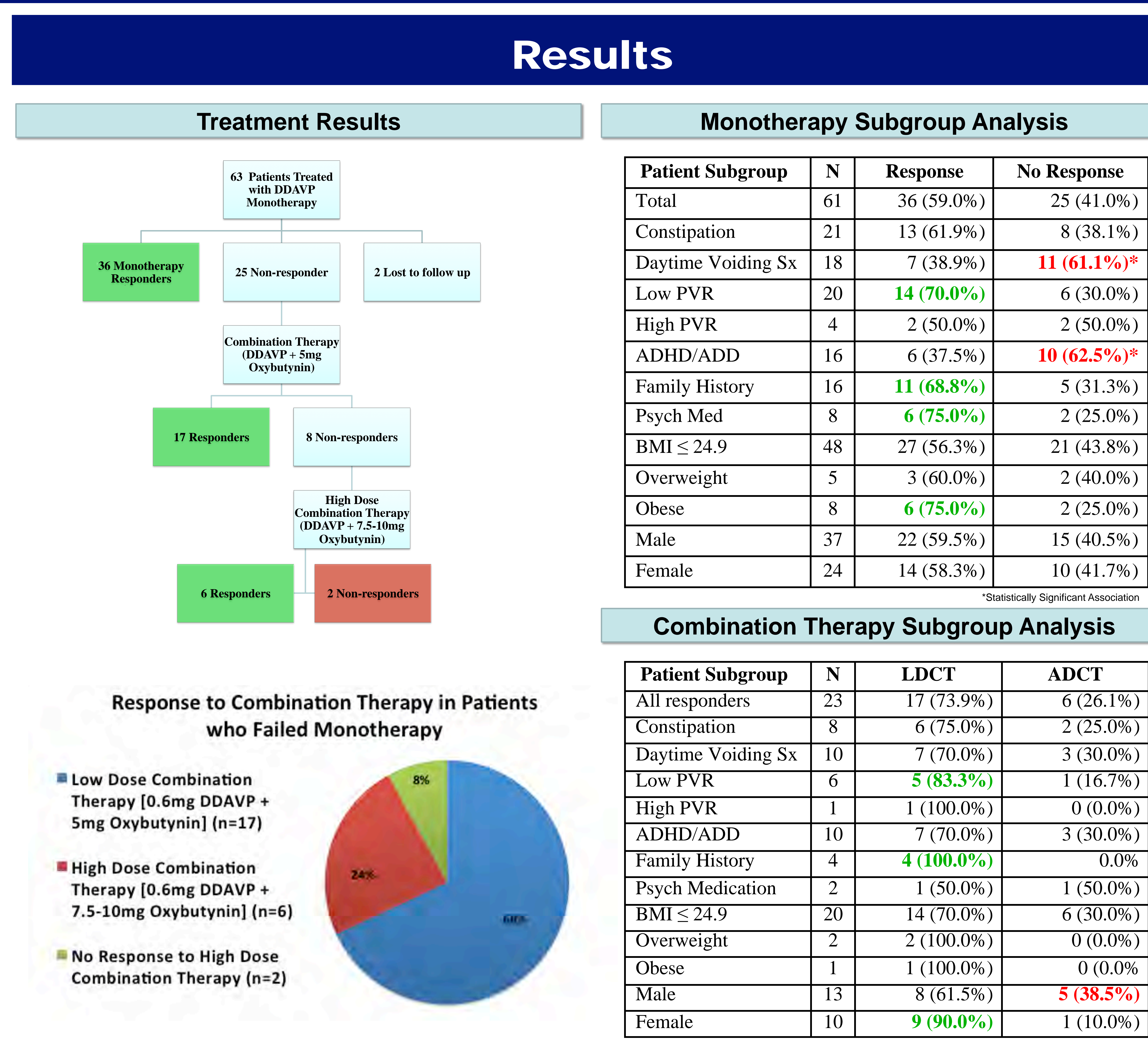

\section{Acknowledgements}

- Family of the late Clifford Gill

- UMMS Department of Urology

- UMMS Department of Pediatric Urology

- Dr. Mitchell Sokoloff

- Dr. Jennifer Yates

- Senior Scholars Faculty and Advisors - Apurv Soni

\section{Conclusions}

$>97 \%$ Overall success rate using dose titration

$>$ No reported adverse events

ADD/ADHD and CDVS* subgroups had statistically significant decreased response to monotherapy

$>$ High monotherapy response rate in low PVR, family History, Psych Medication and Obese subgroups

$>$ Age was not a predictive factor
$>$ High dose combination therapy is safe and effective 